\title{
El Perdón en Relaciones Cercanas: Conceptualización desde una Perspectiva Psicológica e Implicancias para la Práctica Clínica
}

\section{Forgiveness in Close Relationships: Conceptualization from a Psychological Perspective and Implications for the Clinical Practice}

\author{
Mónica Guzmán \\ Universidad Católica del Norte
}

\begin{abstract}
El perdón es un fenómeno encontrado en todas las relaciones humanas, siendo además una temática de amplia presencia en el ámbito psicoterapéutico. Pese a ello, es notoria la escasez de estudios que discutan este tema en Chile. El objetivo del presente artículo es hacer una descripción de la naturaleza de este fenómeno desde una perspectiva psicológica, sistematizando los principales puntos de consenso y disenso en torno a la conceptualización del perdón encontrados en la literatura científica. Se describen además las variables que se han asociado al perdón y algunos instrumentos utilizados para medirlo. Finalmente, se discuten las implicancias clínicas del estudio del perdón y posibles direcciones futuras para la investigación en esta emergente área.
\end{abstract}

Palabras claves: perdón, transgresiones relacionales, medición del perdón.

Forgiveness is a phenomenon found in all human relationships and is widely present in the psychotherapeutic field. However, there is a noticeable absence of studies that discuss this issue in Chile. The aim of this article is to describe the nature of this phenomenon from a psychological perspective, systematizing the main points of consensus and disagreement found in the scientific literature about forgiveness. Also, it describes the factors that have been associated with forgiveness and some instruments to measure it. Finally, clinical implications of forgiveness research are discussed, as well as possible future directions for research in this emerging area.

Keywords: forgiveness, relational transgressions, forgiveness measures.

\section{Introducción}

En todas las relaciones humanas existe la posibilidad de sentirse herido por la conducta del otro, ya sea por cuestiones pequeñas, como algo dicho sin pensar, o más severas, como una traición, situaciones que instalan, de manera más o menos explícita, el tema del perdón.

Este tópico cobra especial relevancia en las relaciones cercanas, como las familiares, de amistad y de pareja, en las que la presencia o ausencia del perdón puede tener implicancias para la calidad de dichos vínculos.

El tema del perdón se presenta también con especial frecuencia en el ámbito psicoterapéutico. Los terapeutas frecuentemente se ven involucrados en conversaciones que guardan relación con sentimientos de dolor y con el perdón en relaciones significativas: si perdonar o no a un padre que ha estado ausente, si perdonar o no a una pareja que ha sido infiel (Legaree, Turner \& Lollis, 2007).

Sin embargo, pese a que la reparación de relaciones dañadas es una de las motivaciones para buscar ayuda psicológica, llama la atención que el perdón no se haya constituido en un foco privilegiado de estudio teórico y empírico, sino solo hasta hace un par de décadas, en que dicho escenario ha cambiado.

Mónica Guzmán G., Escuela de Psicología, Universidad Católica del Norte, Antofagasta, Chile. Doctorado en Psicología, Escuela de Psicología, Pontificia Universidad Católica de Chile, Santiago, Chile.

La correspondencia relativa a este artículo debe ser dirigida a Mónica Guzmán G., Escuela de Psicología, Universidad Católica del Norte, Avenida Angamos 0610, Antofagasta, Chile. E-mail: moguzman@ucn.cl 
Partiendo desde un interés originalmente circunscrito a los ámbitos religioso, antropológico y filosófico, la motivación por el estudio del perdón se ha ido moviendo progresivamente hacia el área de la psicología y, dentro de esta, a la psicología clínica. El interés dentro de este campo se ve reflejado en el creciente número de artículos que describen modelos de intervención tendientes a abordar este tema, especialmente con parejas y familias (Gordon \& Baucom, 2003; McCullough \& Worthington Jr., 1994; Ripley \& Worthington Jr., 2002; Rye \& Pargament, 2002). Dicha motivación se ha fundamentado en dos aspectos. Por un lado, se ha consignado al perdón como un aspecto crítico en el proceso de recuperación de transgresiones mayores, como la infidelidad en el caso de una pareja. El perdón, según algunos autores, sería clave para la comprensión de cómo las personas son capaces de mantener relaciones interpersonales satisfactorias (Allemand, Amberg, Zimprich \& Fincham, 2007; Fincham, Hall \& Beach, 2006; Karremans \& Van Lang, 2004). Por otro lado, un grupo importante de estudios ha recogido evidencia acerca de la asociación entre el perdón y la salud, tanto física como emocional (Bono, McCullough \& Root, 2008; Thompson et al., 2005; Toussiant \& Webb, 2005; Tse \& Yip, 2009; Worthington Jr. \& Scherer, 2004). De hecho, parte del interés por el estudio del perdón se ha enmarcado dentro de la psicología positiva (Casullo, 2008; McCullough \& Witvliet, 2002), corriente que enfatiza el estudio de las virtudes y fortalezas humanas y su incidencia sobre la calidad de vida de las personas (Seligman, 2002).

Pese a este creciente interés por el estudio empírico del perdón en el concierto internacional, se desconocen estudios publicados en Chile que discutan este tópico desde una perspectiva clínica, aun cuando es un tema en el que los terapeutas se ven insoslayablemente involucrados, en la medida en que el trabajo psicoterapéutico se lleva a cabo con el sufrimiento humano, muchas veces asociado a haberse sentido dañado por otro.

Si bien se han publicado en Chile estudios y propuestas teóricas sobre el perdón, estos se han desarrollado en ámbitos como la sociología y la psicología política y social, a partir de los hechos de violencia política vividos en el país y las repercusiones que estos han tenido a nivel colectivo e individual (e.g., Capponi, 1999; Juricic \& Reyes, 1999; Loveman \& Lira, 1999; Manzi \& González, 2007). Sin embargo, no se ha estudiado el perdón en relaciones cercanas ni el impacto que dicha opción tiene tanto en la subjetividad individual como en la calidad de tales relaciones.

En consecuencia, el presente artículo tiene como objetivo sistematizar los resultados de diversas investigaciones en torno al perdón en relaciones cercanas, contribuyendo con ello a la divulgación del conocimiento acumulado en este ámbito. Para ello, se partirá por señalar los acuerdos y disensos existentes en torno a la conceptualización del perdón en la literatura científica, siguiendo con una breve descripción de los factores asociados al mismo, así como de los instrumentos utilizados para medirlo. Finalmente, se discuten algunas de las implicancias que para la práctica clínica conlleva la investigación sobre el perdón y posibles direcciones futuras para su estudio.

Si bien el perdón puede tener como objeto uno mismo, una situación o una persona, el foco de este artículo se concentra en este último. Además, interesa describir el perdón desde la perspectiva de quien se ha sentido herido, es decir, las características y consecuencias de la decisión de perdonar. Por lo mismo, se omiten en este artículo referencias al arrepentimiento y la culpa, por cuanto ellas son vivencias más propias de quien ha ofendido y pide perdón. Del mismo modo, si bien la comprensión cabal del significado psicológico del perdón se enriquece al considerar variables socioculturales, la incorporación de dicho marco escapa a los objetivos de este artículo.

\section{Consensos y Disensos en Torno a la Conceptualización del Perdón}

\section{Acuerdos Relativos en Torno a la Conceptualización del Perdón}

El número de definiciones del perdón en la literatura científica es vasto. Así, por 
ejemplo, Makinen y Johnson (2006) señalan que perdonar involucra reparar heridas emocionales, restaurar la confianza y reconstruir el vínculo. En una visión similar, concebida desde la terapia familiar contextual, Hargrave y Sells (1997) definen el perdón como "un esfuerzo por restaurar el amor y la confianza en las relaciones" (pp. 43).

Por su parte, Gordon y Baucom (1998, 2003) sostienen que el perdón implica una compleja interacción entre quien perdona y quien es perdonado, involucrando el logro de una visión más balanceada de la relación, una disminución de los sentimientos negativos hacia el otro y un menor deseo de castigarlo.

Desde otra perspectiva, Strelan y Covic (2006) conceptualizan el perdón en el marco de los mecanismos de afrontamiento del estrés, definiéndolo como un proceso de neutralización de un estresor que es producto de la percepción de una herida interpersonal.

Finalmente, McCullough, Worthington Jr. y Rachal (1997) conciben el perdón como una transformación motivacional en dos componentes: la motivación a evitar a la persona que ha ofendido y la motivación a buscar venganza, señalando que el perdón implicaría una disminución de ambas motivaciones.

Pese a la diversidad de énfasis de estas definiciones, un primer punto de consenso alude a que la mayoría de los autores coincide en considerar que el perdón implica un descenso en la negatividad de los pensamientos, sentimientos y conductas hacia el ofensor (Fincham et al., 2006; Kachadourian, Fincham \& Davila, 2004, Strelan \& Covic, 2006). Es decir, involucraría una disminución en el resentimiento hacia quien ha provocado el dolor. Además de este punto en común, otro aspecto en el que existe acuerdo es sobre su diferenciación de otros conceptos con los que habitualmente se le confunde. Específicamente, existe consenso en que el perdón debe ser diferenciado de la negación, que implica la falta de disposición para ver que ha ocurrido un daño, el olvido, que supone eliminar de la conciencia la ofensa, y la justificación, que involucra aceptar los motivos por los cuales el ofensor cometió su error (Fincham et al., 2006; Konstam, Chernoff \& Deveney, 2001, Rye, Loiacono, Folck, Olszewski, Heim \& Madia, 2001). Más bien, perdonar implica que la persona que ha vivenciado el dolor de una ofensa reconoce la naturaleza hiriente de esta y, aun a sabiendas de que la situación puede ser injustificada y la persona no merece ser perdonada, decide hacerlo.

De lo anteriormente señalado, se desprende que el perdón implica un proceso en el cual la persona se involucra de manera intencional (Fincham et al., 2006) y que la decisión de perdonar no excluye la opción de reclamar justicia, en la medida en que la motivación de tal reclamo no sea meramente vengativa (Casullo, 2008).

Por otro lado, si bien el perdón sería un cambio que se produce en las propias emociones, pensamientos y conductas, razón por la cual tendría un carácter individual, es también, en otro sentido, interpersonal, dado que se produce dentro de un contexto interaccional específico, en el que los roles ofensor-ofendido suelen ser intercambiables (Hargrave \& Sells, 1997; Hoyt \& McCullough, 2005; Konstam et al., 2001; McCullough, Pargament \& Thoresen, 2000). De hecho, el foco exclusivo sobre la persona herida necesariamente hace referencia a una historia relacional particular con la persona que ha herido. Por lo tanto, el estudio de este fenómeno puede hacerse incorporando explícitamente ambas perspectivas o bien focalizando la atención en una de ellas (ofensor-ofendido), sin que se pierda de vista esta aproximación relacional.

Finalmente, los autores coinciden en que el constructo del perdón puede ser usado y entendido de varias maneras: como una respuesta o estado, una característica de ciertas unidades sociales o una característica de personalidad (McCullough \& Witvliet, 2002). El perdón episódico, o como estado, se refiere al perdón de una ofensa en particular (McCullough et al., 1997; Paleari, Regalia \& Fincham, 2009). El perdón diádico es entendido como la disposición a perdonar dentro de una relación específica, es decir, se conceptualiza como una cualidad de una unidad social en particular (Kachadourian et al., 2004). Así, 
pueden haber unidades sociales, como una pareja o una familia, en las que el perdón es más probable que en otras relaciones. Finalmente, el perdón disposicional es entendido como un rasgo de personalidad que alude a la tendencia a perdonar a través del tiempo y las situaciones (Berry, Worthington Jr., Parrott III, O'Connor \& Wade, 2001; Thompson et al., 2005).

\section{Disensos en Torno a la Conceptualización del Perdón}

Pese a la existencia de elementos comunes en la conceptualización del perdón, continúa siendo tema de debate una serie de aspectos referentes a: la importancia y probable potencial benéfico de perdonar desde un punto de vista clínico; la presencia o no de sentimientos de benevolencia hacia quien ha cometido la ofensa; el momento de término del perdón, esto es, su relación con la reconciliación; y el proceso psicológico mediante el cual se perdona, es decir, los modelos referentes al perdón.

Importancia y probable potencial benéfico del perdón. En una revisión de la literatura sobre el tema, Legaree et al. (2007) discuten cómo las distintas conceptualizaciones del perdón se traducen en diversas prácticas terapéuticas, así como en la asunción, en algunos casos explícita, de ciertas posturas valóricas. Estos autores han descrito tres posturas.

La primera posición, claramente dominante en la literatura, concibe el perdón como crucial para la resolución de heridas y para el bienestar personal, sosteniéndose su importancia para la salud mental y física (e.g., McCullough \& Worthington Jr., 1994; Worthington Jr. \& Drinkard, 2000). El perdón sería efectivo en resolver sentimientos de rabia y ansiedad y sería útil como herramienta clínica para trabajar con una gran variedad de poblaciones, incluyendo personas con estrés postraumático y pacientes con cáncer, entre otros (Konstam et al., 2001). En el ámbito de las relaciones de pareja, los estudios con esta postura indican que la capacidad para perdonar y pedir perdón permite resolver conflictos y aumentar la longevidad y la satisfacción marital
(Feeney, 2004; Fenell, 1993; Fincham et al., 2006; Kachadourian et al., 2004; McCullough et al., 1997; Tsang, McCullough \& Fincham, 2006). Las posturas ideológicas subyacentes a algunos de estos estudios aluden a la perspectiva cristiana y a los valores de compasión y humildad.

Existe una segunda posición, más crítica respecto al perdón. Esta sostiene que no perdonar puede ser también un estado positivo, pues hacerlo podría incluso ser dañino, poniendo en riesgo de re-victimizar a personas que se encuentren en condición de vulnerabilidad por situaciones de abuso o maltrato. Por lo tanto, no lo ven como un mecanismo que necesariamente apunte hacia la salud. Usualmente, estas posturas son asumidas por quienes trabajan con poblaciones víctimas de violencia o injusticias más severas, como el abuso sexual. Los valores que subyacen a esta visión son los de equidad, justicia y empoderamiento (e.g., Bass \& Davis, 1994; Hermann, 1999, ambos citados en Legaree et al., 2007; Kennedy, 2000).

Una tercera postura, intermedia entre las dos anteriores, enfatiza el rol de variables contextuales como indicadores de lo beneficioso que puede ser perdonar, por lo tanto, considera necesario tomar en cuenta el marco relacional en el que este ocurre. La decisión de perdonar o no es de la persona y puede introducirse en el ámbito psicoterapéutico cuando es producto de un elección libre, por lo tanto, no es positivo en sí mismo para todas las personas ni frente a todas las situaciones (Rotter, 2001). El perdón puede aliviar el sufrimiento, pero en otros casos puede exacerbarlo cuando ocurre en un contexto opresivo o ante transgresiones graves que forman parte de un patrón de interacción estable, como es el caso de la violencia. Desde esta postura, no perdonar puede ser tan liberador como hacerlo y es visualizado más bien como una de las alternativas posibles en el proceso de recuperación de heridas relacionales.

Momento de término del perdón. Otra tensión existente alude al momento de término del perdón: si es únicamente la disminución de las respuestas negativas (y el eventual aumento de sentimientos positi- 
vos hacia el ofensor) o bien implica de manera necesaria una reconciliación, entendida como el restablecimiento de la relación. Algunos autores, mayoritarios en número, sostienen que si bien el perdón hace más probable la reconciliación, no desemboca necesariamente en ella: se puede permanecer en una relación sin que haya habido perdón o bien perdonar a alguien con quien ya no se tiene ningún vínculo (e.g., Fincham et al., 2006; Gordon \& Baucom, 2003). Otros autores, menores en número, sostienen que la reconciliación es una parte definitoria del perdón (e.g., Hargrave \& Sells, 1997).

Carácter unidimensional o bidimensional del perdón. En estrecha vinculación al punto anterior, se discute si el perdón tiene un carácter unidimensional, es decir, si solo involucra un descenso en la negatividad de las emociones, pensamientos y conductas hacia el ofensor o si incluye, además, una dimensión de benevolencia, esto es, el desarrollo de sentimientos de compasión, empatía o incluso amor hacia el ofensor.

La formulación inicial de McCullough, Rachal, Sandage, Worthington Jr., Brown y Hight (1998), ampliamente utilizada en las investigaciones empíricas, propone una concepción unidimensional: el descenso en la motivación a evitar y a vengarse del ofensor serían suficientes para considerar que alguien ha perdonado a otro. Del mismo modo, Gordon y Baucom $(1998,2003)$ señalan que el perdón no requiere una orientación positiva hacia el otro, pues puede ser algo que se realiza para sí mismo.

Sin embargo, otros autores señalan que el perdón incluye necesariamente una dimensión de benevolencia (e.g., Hargrave \& Sells, 1997; Human Development Study Group, University of Wisconsin, Madison, 1991), posición a la cual se han ido sumando otros autores, como McCullough, Root, Tabak y Witvliet (2009), quienes recientemente han agregado los sentimientos positivos a la definición del mismo. Sin embargo, ante esta última posición surge la cuestión de qué sucede en aquellas relaciones que no continúan: ¿el perdón incluye necesariamente sentimientos positivos hacia alguien con quien no se tiene ningún vínculo o cercanía afectiva? Fincham et al. (2006), situados probablemente en una posición intermedia, sostienen que el perdón contendría elementos positivos solo en relaciones significativas y/o que se mantienen.

Modelos respecto al perdón. Existe una variedad importante de formulaciones acerca de cómo ocurre el perdón. La mayor parte de estos planteamientos tiene a la base la hipótesis de que perdonar, más que un acto único, sería un proceso y que, como tal, se desarrolla en el tiempo. En una revisión hecha por Strelan y Covic (2006) se menciona la existencia de gran cantidad de modelos; no obstante, solo unos pocos han sido sometidos a validación empírica (e.g., Gordon \& Baucom, 1998, 2003; Hargrave \& Sells, 1997; Pollard, Anderson, Anderson \& Jennings, 1998).

Entre las propuestas validadas empíricamente, a modo de ejemplo se puede señalar el modelo de Hargrave y Sells (1997), constituido por dos componentes: la exoneración y el perdón propiamente tal. La exoneración sería un proceso interno en que la persona alcanza mayor comprensión de la situación evocadora de dolor. El perdón, en cambio, tendría un carácter más interpersonal, en el cual ofensor y ofendido se involucran en interacciones que apuntan hacia el restablecimiento del amor y la confianza. En esta etapa se requiere dar la oportunidad para la compensación y realizar actos abiertos de perdón.

Por su parte, Gordon y Baucom (1998, 2003) proponen un modelo del perdón específico para las parejas, aplicado fundamentalmente a transgresiones severas. Está dividido en tres etapas: impacto, en el cual la persona toma conciencia del efecto de la herida; significación, que implica descubrir el sentido de lo ocurrido; y recuperación, que involucra elaborar un nuevo set de creencias relacionales.

Los modelos del perdón se han ocupado en el diseño de intervenciones en el ámbito psicoterapéutico y educativo. La mayor parte de estos programas de intervención se han diseñado más para grupos que para individuos. Algunos han tenido un foco claramente clínico (e.g., Reed \& Enright, 2006; Rye \& Pargament, 2002), mientras otros han apuntado más bien 
hacia la prevención y la educación (e.g., Lampton, Oliver, Worthington Jr. \& Berry, 2005). Pese a esta diversidad, los modelos de intervención que se han sometido a validación empírica han sido más eficaces que el no tratamiento (para una revisión detallada de las intervenciones ver Lundahl, Taylor, Stevenson \& Roberts, 2008).

Frente a la proliferación de diversos modelos que han mostrado ser eficaces, McCullough y Worthington Jr. (1994) sugieren que, en lugar de existir un único modelo del perdón con carácter nomotético, habría distintas propuestas válidas, toda vez que permitirían explicar las diferencias individuales en el perdón.

\section{Factores Asociados al Perdón}

Para perdonar debe haber existido una ofensa y esta se da necesariamente inserta en un contexto; por lo tanto, la comprensión del perdón como fenómeno complejo requiere conocer aquellas variables asociadas al mismo.

Entre los factores que han mostrado relación con el perdón, se encuentran variables relacionadas con el que perdona, la ofensa, el ofensor y la relación (Hoyt \& McCullough, 2005), además de características sociodemográficas.

En relación a las características del que perdona, se ha encontrado consistentemente que hay mayor disposición a perdonar cuando existen atribuciones benignas respecto de la conducta del otro (Fincham, Jackson \& Beach, 2005; Hall \& Fincham, 2006; Jackson, 2002) y cuando se tiene empatía hacia el ofensor (Berry et al., 2005; Edwards, 2007; Hodgson \& Wertheim, 2007; Rizkalla, Wertheim \& Hodgson, 2008).

También se ha visto que el perdón estaría asociado a la habilidad para manejar las emociones: las personas que experimentan mayor afecto negativo, como ansiedad o depresión, perdonan menos (Hodgson \& Wertheim, 2007; Lawler-Row, Younger, Piferi \& Jones, 2006; Worthington Jr. \& Wade, 1999).

Del mismo modo, la disposición a perdonar está asociada directamente a la agradabilidad e inversamente tanto al narcisismo (Eaton, Struthers \& Santelli, 2006) como al neuroticismo (Brose, Rye, Lutz-Zois \& Ross, 2005). Respecto a este último punto, se ha propuesto que la relación inversa entre ambas variables podría ser explicada porque las transgresiones se vivenciarían como más severas (McCullough \& Hoyt, 2002).

Desde otra perspectiva, una de las formulaciones teóricas que se ha incorporado al estudio del perdón es la teoría del apego (Bowlby, 1980). Los estudios han mostrado de manera consistente que las personas con estilos de apego seguro tienen mayor disposición a perdonar que las personas con estilos de apego inseguro (Burnette, Davis, Green, Worthington Jr. \& Bradfield, 2009; Edwards, 2007; Finkel, Burnette \& Scissors, 2007; Lawler-Row et al., 2006, Vuncannon, 2006; Webb, Call, Chickering, Colburn \& Heisler, 2006).

En relación a las características de la ofensa, se ha observado que el perdón sería menos probable mientras más severa es evaluada aquella (Fincham et al., 2005; Rye \& Pargament, 2002).

En cuanto a las características del ofensor, se ha encontrado que la humildad y las disculpas sinceras ofrecidas por quien ha ofendido harían más probable el perdón (Bachman \& Guerrero, 2006; Fincham et al., 2005; McCullough et al., 1997; Wade \& Worthington Jr., 2003; Zechmeister, Garcia, Romero \& Vas, 2004).

Por otra parte, tres son las variables más investigadas dentro de los factores de la relación asociados al perdón: cercanía, satisfacción y compromiso. Se ha visto que el perdón es más probable que ocurra cuando hay un alto grado de cercanía con la pareja (Tsang, McCullough \& Fincham, 2006), cuando la satisfacción con la relación es alta (Allemand et al., 2007; Kachadourian et al., 2004) y cuando hay un alto nivel de compromiso con ella (Cann \& Baucom, 2004; McCullough et al., 1998). Además, Finkel, Rusbult, Kumashiro y Hannon (2002) encontraron que la relación entre compromiso y perdón estaba mediada por la interpretación cognitiva de las transgresiones.

Finalmente, en cuanto a las características sociodemográficas, la variable que ha mostrado la relación más consistente con el perdón es el sexo. Diversos estudios 
muestran que las mujeres perdonarían más que los hombres (e.g., Finkel et al., 2002; Orathinkal, Vansteenwegen \& Burggraeve, 2008), lo cual es reforzado por la revisión meta-analítica llevada a cabo por Miller, Worthington Jr. y McDaniel (2008). También se ha encontrado que la tendencia a perdonar se incrementa con la edad (Girard \& Mullet, 1997; Park \& Enright, 1997), lo que podría tener vinculación con el desarrollo moral y cognitivo (McCullough \& Witvliet, 2002). Del mismo modo, se ha visto que la presencia de hijos está asociada a mayor perdón (e.g., Orathinkal, et al., 2008).

Pese a estos resultados, no ha habido suficiente consistencia en los hallazgos, salvo en el caso de las diferencias por sexo, requiriéndose más investigaciones que exploren posibles variaciones en el perdón según características sociodemográficas.

La mayor parte de estos estudios ha investigado estos predictores de manera separada, siendo menos los que han examinado el perdón como un fenómeno complejo, multideterminado, que se comprende a partir de la interacción entre una serie de variables. A modo de ejemplo, es posible mencionar el estudio desarrollado por Edwards (2007), quien encontró que la seguridad del apego estaba asociada a mayor perdón y que dicha relación estaba mediada parcialmente por sentimientos más constructivos hacia el conflicto y mayor empatía hacia el agresor. En un esfuerzo similar, en el estudio de Burnette et al. (2009) se confirmó la asociación entre la seguridad del apego y el perdón, encontrándose además que dicha relación estaba mediada por la excesiva tendencia a rumiar acerca de la transgresión (rumination) en personas con alta ansiedad del apego y por la falta de empatía en personas con alta evitación del apego.

\section{Medición del Perdón}

En la actualidad existe una serie de instrumentos que evalúan el perdón, los que pueden ser categorizados de acuerdo al tipo de perdón que miden y a las técnicas de evaluación. El primer aspecto, tipo de perdón, está representado por la especificidad de la medición, es decir, si se está evaluando el perdón episódico, diádico o disposicional.

A modo de ejemplo, a continuación se mencionan algunos de los instrumentos más utilizados en la investigación empírica y que poseen además buenas propiedades psicométricas.

Entre los instrumentos que evalúan el perdón episódico, se encuentra el Transgression-Related Interpersonal Motivations Inventory (TRIM-12, McCullough et al., 1998), uno de los instrumentos más utilizados en los estudios en esta área, por su brevedad y buenas propiedades psicométricas. Este inventario evalúa dos dimensiones, la motivación a buscar venganza y la motivación a evitar al ofensor. El TRIM-12 tiene adecuada consistencia interna, medida con alfa de Cronbach $(\alpha=0,86$ para la escala de evitación y $\alpha=0,90$ para la escala de venganza). Además, posee una apropiada validez, demostrada por su asociación con la medición del perdón mediante un ítem único $(p<0,01)$, tanto en estudiantes universitarios que habían experimentado una transgresión recientemente como en quienes manifestaron su interés en participar en una intervención que los ayudase a perdonar a una persona que los había herido en el pasado. Del mismo modo, las escalas del TRIM han mostrado asociación con variables de la relación, tales como la satisfacción, el compromiso y la cercanía ( $p$ $<0,01$ ) (McCullough et al., 1998). Recientemente, se ha agregado al instrumento la dimensión de benevolencia (McCullough, Root \& Cohen, 2006).

En cuanto a los instrumentos que han medido el perdón diádico, se encuentra la Interpersonal Resolution Scale (IRRS, Hargrave \& Sells, 1997), compuesta por 44 ítems, divididos en dos escalas (perdón y dolor), que evalúan en qué medida se ha perdonado a una persona dentro de una relación específica, típicamente un miembro de la familia, con quien se ha tenido una historia de transgresiones relacionales. Basados en un procedimiento de cinco etapas, los autores reportan una adecuada validez de constructo del instrumento, demostrada por su correlación con otros aspectos del funcionamiento familiar (e.g., autoridad personal e intimidación intergeneracional) en una muestra de población normal $(p<$ 
0,01). En términos de su validez predictiva, el instrumento discriminó entre una muestra clínica y una no clínica que habían experimentado algún tipo de transgresión en su familia. Las personas de la muestra clínica reportaron niveles significativamente más bajos de perdón que las personas de la muestra no clínica $(p<0,01)$. Finalmente, los índices de consistencia interna, medida mediante alfa de Cronbach, son 0,92 y 0,95 para las escalas de perdón y dolor, respectivamente.

Finalmente, entre los instrumentos que miden el perdón disposicional, se encuentra el Transgression Narrative Test of Forgivingness (TNTF, Berry et al., 2001). Este es un instrumento compuesto por cinco escenarios ficticios en los que la persona debe indicar con qué probabilidad perdonaría la situación descrita en cada viñeta. Además de su brevedad, se caracteriza por su relativa neutralidad teórica. Respecto de sus propiedades psicométricas, Berry et al. (2001) condujeron una serie de cinco estudios con estudiantes universitarios en los que se encontró que la escala estaba correlacionada inversamente con la tendencia a rumiar, la rabia crónica, la hostilidad y el neuroticismo $(p<0,01)$ y directamente con la agradabilidad $(p<0,01)$. Además, reportaron que los índices de consistencia interna, evaluada mediante alfa de Cronbach, fluctuaron entre 0,73 y 0,81 .

En cuanto a las técnicas para evaluar perdón, la mayoría de los instrumentos disponibles son cuestionarios de auto-reporte, ampliamente utilizados en las investigaciones, pero que poseen la desventaja de tener sesgos asociados, tales como la deseabilidad social y la aquiescencia.

$\mathrm{Al}$ respecto y a fin de aumentar la validez de la investigación acerca del perdón, Hoyt y McCullough (2005) proponen la medición multimodal del perdón, esto es, la incorporación de diferentes técnicas, por ejemplo, eventos de la vida, datos observacionales, reporte de terceros, además de los auto-reportes, así como diferentes niveles de análisis (perdón episódico, diádico y disposicional).

Por otro lado, McCullough et al. (2009) señalan que además de las mediciones del perdón en un único momento (diseños trans- versales), el perdón puede ser medido como un proceso que se desarrolla en el tiempo, mediante estudios longitudinales, que son diseños que se ajustarían mejor a una conceptualización dinámica del perdón.

Finalmente, es importante consignar que en Chile no existen estudios de adaptación o validación de estos instrumentos. Un paso adelante en este tema es la investigación que se encuentra en marcha para realizar la adaptación del TRIM-12 al español.

\section{Discusión}

El perdón, tal como se lo ha descrito en la presente sistematización, puede ser concebido como un constructo psicológico, pero también como una opción terapéutica para trabajar con el impacto que a corto y largo plazo provoca una transgresión relacional. Entendido como tal, se estima que el desarrollo de investigaciones acerca del perdón tiene una serie de implicancias para la práctica clínica.

En primer lugar, permite generar reflexión y discusión respecto de un fenómeno que está de todas maneras presente en la vida cotidiana $\mathrm{y}$, por ende, también en el ámbito psicoterapéutico. Integrar la investigación sobre el perdón da espacio a una práctica clínica respaldada empíricamente, reduciendo con ello el riesgo de implementar intervenciones basadas solo en concepciones personales o intuitivas respecto del mismo.

Así, por ejemplo, los hallazgos en torno a este tema ofrecen una guía para decidir cuándo, cómo y bajo qué condiciones podría ser beneficioso instalar una intervención orientada al perdón. Si bien hay amplio respaldo a la noción de que el perdón favorecería la salud emocional y física, no se puede afirmar que este traerá consecuencias positivas para todos los consultantes o en todas las situaciones. En lo psicoterapéutico, por lo tanto, se considera necesario evaluar caso a caso la pertinencia de implementar una intervención de este tipo, tomando en cuenta que la decisión de trabajar o no en este es producto de una co-construcción entre paciente y terapeuta.

Tal propuesta se fundamenta en el hecho de que ni aun los autores que adhieren 
a la noción de que perdonar es beneficioso adoptan una postura generalista. Hacerlo sería sobre simplificar un tema que es de por sí complejo. Se estima que la concepción del perdón como una estrategia de afrontamiento, entre otras posibles, permite zanjar la discusión en torno al potencial benéfico del perdón: su conveniencia dependerá más bien de variables individuales y contextuales

En la misma línea, la investigación en torno a los límites del concepto (qué es y qué no es el perdón) es de gran importancia para la práctica clínica, por cuanto las personas que van a terapia y los terapeutas, por cierto, poseen sus propias concepciones o teorías respecto a lo que es el perdón y cómo alcanzarlo. No tener dicha claridad, o al menos no compartir explícitamente dichas concepciones para alcanzar una definición consensuada, podría aumentar la probabilidad de hacer intervenciones iatrogénicas: por ejemplo, si se hace equivalente el perdón a reconciliación, se puede incrementar el riesgo de que una persona se exponga a seguir siendo dañada por otro.

Del mismo modo, se considera que la discusión respecto a la dimensionalidad del perdón también es relevante para la psicoterapia, es decir, si este involucra necesariamente el desarrollo de sentimientos de benevolencia o si es suficiente con dejar de experimentar emociones negativas hacia el ofensor. ¿Basta con intervenir para que el resentimiento disminuya y la persona se libere de las emociones negativas que la aquejan o es necesario, además, promover que esta desarrolle sentimientos positivos hacia quien la ha herido? Este es un tema en el que los autores todavía no han llegado a consenso. Pareciera ser que una forma de resolver el asunto sería que la disminución de emociones negativas y el aumento de emociones positivas serían independientes y que esta última dimensión podría ser necesaria en relaciones significativas que continúan, pero probablemente no sea condición sine qua non en vínculos ya disueltos.

La investigación que se ha desarrollado hasta ahora ha permitido responder una serie de interrogantes respecto del perdón. Pero también, conforme avanza el estado del conocimiento en este ámbito, surgen nuevas preguntas y desafíos que necesitan ser abordados en futuros estudios. En primer lugar, se ha acumulado un cuerpo de conocimiento considerable en torno al hecho de perdonar, es decir, desde la postura de quien se ha sentido herido. Sin embargo, se sabe bastante menos de la perspectiva de quien ha ofendido y lo que sucede con la persona cuando es perdonada por otra. Se hace necesario integrar en estudios futuros esta mirada, dado que en la vida cotidiana las transgresiones suelen ser mutuas e, incluso en una misma situación, una persona puede desempeñar ambos roles: ofensor y ofendido. ¿Qué favorece que una persona se arrepienta y pida perdón? ¿Cómo es la vivencia de ser perdonado por otro?

Segundo, se considera necesario investigar más en profundidad las variaciones que puedan existir en la dinámica del perdón en los distintos tipos de relaciones: ¿es similar la dinámica del perdón en una relación padre-hijo, en una relación de amistad o en una relación de pareja? Falta desarrollar estudios que específicamente aborden este tema.

Tercero, dado que los factores que pueden favorecer o inhibir al perdón son distintos en cada situación, se considera absolutamente necesario integrar dichos elementos en el estudio del perdón, tanto para comprenderlo como para establecer su valor benéfico. Así, por ejemplo, perdonar una crítica ocurrida en una única oportunidad es distinto y trae diferentes consecuencias que perdonar la crítica constante que forma parte de un patrón de interacción estable.

Cuarto, es importante tener presente que en la conceptualización del perdón, quizás más que en otros temas, ejercen influencia variables de tipo religioso, cultural e ideológico-político y, dado que la investigación no se da con independencia de dicho contexto, es necesario explicitar desde qué marco se está trabajando cuando se implementan intervenciones clínicas sobre el perdón.

Quinto, se requiere realizar más estudios que permitan capturar la naturaleza compleja del perdón, proponiendo modelos teóricos que den cuenta de la interrelación de variables de distintos niveles: de la persona herida, del ofensor, de la relación, de 
la ofensa. Estudios de este tipo constituirían un aporte al desarrollo de esta área, especialmente en cuanto a sus implicancias psicoterapéuticas. Así, por ejemplo, saber qué variables podrían mediar la relación entre apego y perdón permitirá distinguir y diseñar focos de intervención más específicos, con énfasis en aquellas variables mediadoras.

Sexto, el campo además podría beneficiarse de estudios experimentales o con diseños longitudinales que permitan establecer influencias causales, los que han sido minoritarios en relación a los diseños transversales y correlacionales.

Séptimo, si se desea adoptar la perspectiva del perdón como un proceso en etapas, es de suma relevancia que las diversas formulaciones o modelos respecto del perdón sean sometidos a evaluación empírica, porque es en base a ellos que pueden diseñarse programas de intervención aplicables en el ámbito clínico.

Se estima que en la elaboración de tales modelos la integración de metodologías cualitativas podría enriquecer el área, conociendo cómo conceptualiza la gente común el perdón. Al ser un tema respecto del cual la mayoría de las personas tiene experiencia, integrar sus propias concepciones podría generar resultados con mayor validez ecológica.

Finalmente, en un tema que es tan sensible a cuestiones culturales e ideológicas, se hace más necesario aún desarrollar investigaciones que busquen comprender el perdón desde una perspectiva clínica contextualizada a la propia realidad, en este caso, la chilena. En el concierto internacional ya se ha asistido a un crecimiento explosivo de estudios sobre el tema; falta que se abra ese espacio en Chile. El desafío está presente.

\section{Referencias}

Allemand, M., Amberg, I., Zimprich, D. \& Fincham, F. D. (2007). The role of trait forgiveness and relationship satisfaction in episodic forgiveness. Journal of Social \& Clinical Psychology, 26, 199217.

Bachman, G. F. \& Guerrero, L. K. (2006). Forgiveness, apology, and communicative responses to hurtful events. Communication Reports, 19, 45-56.

Berry, J. W., Worthington Jr., E. L., Parrot III,
L., O’Connor, L. E. \& Wade, N. G. (2001). Dispositional forgivingness: Development and construct validity of the Transgression Narrative Test of Forgivingness (TNTF). Personality and Social Psychology Bulletin, 27, 1277-1290.

Bono, G., McCullough, M. E. \& Root, L. M. (2008). Forgiveness, feeling connected to others, and well-being: Two longitudinal studies. Personality and Social Psychology Bulletin, 34, 182-195.

Bowlby, J. (1980). La pérdida afectiva. Buenos Aires: Paidós.

Brose, L. A., Rye, M. S., Lutz-Zois, C. \& Ross, S. R. (2005). Forgiveness and personality traits. Personality and Individual Differences, 39, 3546.

Burnette, J. L., Davis, D. E., Green, J. D., Worthington Jr., E. L. \& Bradfield, E. (2009). Insecure attachment and depressive symptoms: The mediating role of rumination, empathy, and forgiveness. Personality and Individual Differences, 46, 276-280.

Cann, A. \& Baucom, T. R. (2004). Former partners and new rivals as threats to a relationship: Infidelity type, gender, and commitment as factors related to distress and forgiveness. Personal Relationships, 11, 305-318.

Capponi, R. (1999). Chile: un duelo pendiente. Perdón, reconciliación, acuerdo social. Santiago, Chile: Editorial Andrés Bello.

Casullo, M. (2008). La capacidad para perdonar. En M. Casullo (Ed.), Prácticas en psicología positiva (pp. 39-60). Buenos Aires: Lugar Editorial.

Eaton, J., Shruthers, C. W. \& Santelli, A. G. (2006). Dispositional and state forgiveness: The role of self-esteem, need for structure, and narcissism. Personality and Individual Differences, 41, 371380.

Edwards, J. K. (2007). Relationship satisfaction: The role of attachment, conflict, empathy and forgiveness. Tesis no publicada para optar al grado de Doctor en Filosofía, Purdue University, West Lafayette, IN, Estados Unidos.

Feeney, J. A. (2004). Hurt feelings in couple relationships: Towards integrative models of the negative effects of hurtful events. Journal of Social and Personal Relationships, 21, 487-508.

Fenell, D. L. (1993). Characteristics of long-term first marriages. Journal of Mental Health Counseling, 15, 446-460.

Fincham, F. D., Hall, J. \& Beach, S. R. H. (2006). Forgiveness in marriage: Current status and future directions. Family Relations, 55, 415-427.

Fincham, F. D., Jackson, H. \& Beach, S. R. H. (2005). Transgression severity and forgiveness: Different moderators for objective and subjective severity. Journal of Social \& Clinical Psychology, 24, 860875.

Finkel, E. J., Burnette, J. L. \& Scissors, L. E. (2007). Vengefully ever after: Destiny beliefs, state attachment anxiety, and forgiveness. Journal of Personality and Social Psychology, 92, 871-886.

Finkel, E. J., Rusbult, C. E., Kumashiro, M. \& Hannon, P. A. (2002). Dealing with betrayal in close relationships: Does commitment promote forgiveness? Journal of Personality and Social Psychology, 82, 956-974. 
Girard, M. \& Mullet, E. (1997). Forgiveness in adolescents, young, middle-aged, and older adults. Journal of Adult Development, 4, 209-220.

Gordon, K. C. \& Baucom, D. H. (1998). Understanding betrayals in marriage: A synthesized model of forgiveness. Family Process, 37, 425-449.

Gordon, K. C. \& Baucom, D. H. (2003). Forgiveness and marriage: Preliminary support for a measure based on a model of recovery from a marital betrayal. The American Journal of Family Therapy, 31, 179-199.

Hall, J. H. \& Fincham, F. D. (2006). Relationship dissolution following infidelity: The roles of attributions and forgiveness. Journal of Social \& Clinical Psychology, 25, 508-522.

Hargrave, T. D. \& Sells, J. N. (1997). The development of a forgiveness scale. Journal of Marital \& Family Therapy, 23, 41-62.

Hodgson, L. K. \& Wertheim, E. H. (2007). Does good emotion management aid forgiving? Multiple dimensions of empathy, emotion management and forgiveness of self and others. Journal of Social and Personal Relationships, 24, 931-949.

Hoyt, W. T. \& McCullough, M. E. (2005). Issues in the multimodal measurement of forgiveness. En E. L. Worthington Jr. (Ed.), Handbook of forgiveness (pp. 207-226). New York: Brunner-Routledge.

Human Development Study Group, University of Wisconsin, Madison (1991). Five points on the construct of forgiveness within psychotherapy. Psychotherapy Theory, Research, Practice, Training, 28, 493-496.

Jackson, M. (2002). The role of rejection sensitivity, attachment, and attributions in forgiveness of romantic partners. Tesis no publicada para optar al grado de Master en Ciencias, University of Georgia, Athens, GA, Estados Unidos.

Juricic, M. F. \& Reyes, M. J. (1999). El sí-no de la reconciliación nacional. Las representaciones sociales de la reconciliación nacional en los jóvenes. Memoria para optar al título de Psicólogo, Escuela de Psicología, Universidad de Chile, Santiago, Chile.

Kachadourian, L. K., Fincham, F. \& Davila, J. (2004). The tendency to forgive in dating and married couples: The role of attachment and relationship satisfaction. Personal Relationships, 11, 373-393.

Karremans, J. C. \& Van Lang, P. A. M. (2004). Back to caring after being hurt: The role of forgiveness. European Journal of Social Psychology, 34, 207. 227.

Kennedy, M. (2000). Christianity and child sexual abuse: The survivors' voice leading to change. Child Abuse Review, 9, 124-141.

Konstam, V., Chernoff, M. \& Deveney, S. (2001). Toward forgiveness: The role of shame, guilt, anger, and empathy. Counseling and Values, 46(3), 26-39.

Lampton, C., Oliver, G. J., Worthington Jr., E. L. \& Berry, J. W. (2005). Helping Christian college students become more forgiving: An intervention study to promote forgiveness as part of a program to shape Christian character. Journal of Psychology \& Theology, 33, 278-290.

Lawler-Row, K. A., Younger, J. W., Piferi, R. L. \& Jones, W. H. (2006). The role of adult attachment style in forgiveness following an interpersonal offense. Journal of Counseling and Development, 84, 493-502.

Legaree, T. A., Turner, J. \& Lollis, S. (2007). Forgiveness and therapy: A critical review of conceptualizations, practices, and values found in the literature. Journal of Marital \& Family Therapy, 33,192-213.

Loveman, B. \& Lira, E. (1999). Las suaves cenizas del olvido: vía chilena de reconciliación política 18141932. Santiago, Chile: LOM Ediciones.

Lundahl, B. W., Taylor, M. J., Stevenson, R. \& Roberts, K. D. (2008). Process-based forgiveness interventions: A meta-analytic review. Research on Social Work Practice, 18, 465-478.

Makinen, J. A. \& Johnson, S. M. (2006). Resolving attachment injuries in couples using emotionally focused therapy: Steps toward forgiveness and reconciliation. Journal of Consulting and Clinical Psychology, 74, 1055-1064.

Manzi, J. \& González, R. (2007). Forgiveness and reparation in Chile: The role of cognitive and emotional intergroup antecedents. Peace and Conflict: Journal of Peace Psychology, 13, 71-91.

McCullough, M. E. \& Hoyt, W. T. (2002). Transgressionrelated motivational dispositions: Personality substrates of forgiveness and their links to the Big Five. Personality and Social Psychology Bulletin, 28, 1556-1573.

McCullough, M. E., Pargament, K. I. \& Thoresen, C. E. (2000). The psychology of forgiveness: History, conceptual issues, and overview. En M. E. McCullough, K. I. Pargament \& K. L. Thoresen (Eds.), Forgiveness: Theory, research, practice (pp. 1-16). New York: Guilford Press.

McCullough, M. E., Rachal, K. C., Sandage, S. J., Worthington Jr., E. L., Brown, S. W. \& Hight, T. L. (1998). Interpersonal forgiving in close relationships: II. Theoretical elaboration and measurement. Journal of Personality and Social Psychology, 75, 1586-1603.

McCullough, M. E., Root, L. M. \& Cohen, A. D. (2006). Writing about the benefits of an interpersonal transgression facilitates forgiveness. Journal of Consulting and Clinical Psychology, 74, 887-897.

McCullough, M. E., Root, L. M., Tabak, B. \& Witvliet, C. V. O. (2009). Forgiveness. En C. R. Snyder \& S. J. Lopez (Eds.), Handbook of positive psychology ( $2^{\text {nd }}$ ed., pp. 427-435). New York: Oxford University Press.

McCullough, M. E. \& Witvliet, C. V. O. (2002). The psychology of forgiveness. En C. R. Snyder \& S. J. Lopez (Eds.), Handbook of positive psychology $\left(2^{\text {nd }}\right.$ ed., pp. 446-455). New York: Oxford University Press.

McCullough, M. E. \& Worthington Jr., E. L. (1994). Models of interpersonal forgiveness and their application to counseling: A review and critique. Counseling and Values, 39(1), 2-14.

McCullough, M. E., Worthington Jr., E. L. \& Rachal, K. C. (1997). Interpersonal forgiving in close relationships. Journal of Personality and Social Psychology, 73, 321-336.

Miller, A. J., Worthington Jr., E. L. \& McDaniel, M. A. (2008). Gender and forgiveness: A meta-analytic review and research agenda. Journal of Social \& 
Clinical Psychology, 27, 843-876.

Orathinkal, J., Vansteenwegen, A. \& Burggraeve, R. (2008). Are demographics important for forgiveness? The Family Journal: Counseling and Therapy for Couples and Families, 16, 20-27.

Paleari, F. G., Regalia, C. \& Fincham, F. D. (2009). Measuring offence-specific forgiveness in marriage: The Marital Offence-Specific Forgiveness Scale (MOFS). Psychological Assessment, 21, 194209.

Park, Y. O. \& Enright, R. D. (1997). The development of forgiveness in the context of adolescent friendship conflict in Korea. Journal of Adolescence, 20, 393402.

Pollard, M. W., Anderson, R. A., Anderson, W. T. \& Jennings, G. (1998). The development of a family forgiveness scale. Journal of Family Therapy, 20, 95-109.

Reed, G. L. \& Enright, R. D. (2006). The effects of forgiveness therapy on depression, anxiety, and posttraumatic stress for women after spousal emotional abuse. Journal of Consulting and Clinical Psychology, 74, 920-929.

Ripley, J. S. \& Worthington Jr., E. L. (2002). Hopefocused and forgiveness-based group interventions to promote marital enrichment. Journal of Counseling and Development, 80, 452-463.

Rizkalla, L., Wertheim, E. H. \& Hodgson, L. K. (2008). The roles of emotion management and perspective taking in individuals' conflict management styles and disposition to forgive. Journal of Research in Personality, 42, 1594-1601.

Rotter, J. C. (2001). Letting go: Forgiveness in counseling. The Family Journal: Counseling and Therapy for Couples and Families, 9, 174-177.

Rye, M. S., Loiacono, D. M., Folck, C. D., Olszewski, B. T., Heim, T. A. \& Madia, B. P. (2001). Evaluation of the psychometric properties of two forgiveness scales. Current Psychology: Developmental, Learning, Personality, Social, 20, 260-277.

Rye, M. S. \& Pargament, K. I. (2002). Forgiveness and romantic relationships in college: Can it heal the wounded heart? Journal of Clinical Psychology, 58, 419-441.

Seligman, M. E. P. (2002). Positive psychology, positive prevention, and positive therapy. En C. R. Snyder \& S. J. Lopez. (Eds.), Handbook of positive psychology ( $2^{\text {nd }}$ ed., pp. 3-12). New York: Oxford University Press.

Strelan, P. \& Covic, T. (2006). A review of forgiveness process models and a coping framework to guide future research. Journal of Social \& Clinical Psychology, 25, 1059-1085.

Fecha de recepción: Julio de 2009.

Fecha de aceptación: Noviembre de 2009.
Thompson, L. Y., Snyder, C. R., Hoffman, L., Michael, S. T, Rasmussen, H. N., Billings, L. S. et al. (2005). Dispositional forgiveness of self, others, and situations. Journal of Personality, 73, 313360.

Toussaint, L. \& Webb, J. R. (2005). Theoretical and empirical connections between forgiveness, mental health, and well-being. En E. L. Worthington Jr. (Ed.), Handbook of forgiveness (pp. 207-226). New York: Brunner-Routledge.

Tsang, J. A., McCullough, M. E. \& Fincham, F. D. (2006). The longitudinal association between forgiveness and relationship closeness and commitment. Journal of Social \& Clinical Psychology, 25, 448-472.

Tse, W. S. \& Yip, T. H. J. (2009). Relationship among dispositional forgiveness of others, interpersonal adjustment and psychological well-being: Implication for interpersonal theory of depression. Personality and Individual Differences, 46, 365368.

Vuncannon, J. (2006). The perceptions of attachment styles and forgiveness in romantic couples. Tesis no publicada para optar al grado de Doctor en Filosofía, Regent University, Virginia Beach, VA, Estados Unidos.

Wade, N. G. \& Worthington Jr., E. L. (2003). Overcoming interpersonal offenses: Is forgiveness the only way to deal with unforgiveness? Journal of Counseling and Development, 81, 343-353.

Webb, M., Call, S., Chickering, S. A., Colburn, T. A. \& Heisler, D. (2006). Dispositional forgiveness and adult attachment styles. The Journal of Social Psychology, 146, 509-512.

Worthington Jr., E. L. \& Drinkard, D. T. (2000). Promoting reconciliation through psychoeducational and therapeutic interventions. Journal of Marital \& Family Therapy, 26, 93-101.

Worthington Jr., E. L. \& Scherer, M. (2004). Forgiveness as an emotion-focused coping strategy that can reduce health risks and promote health resilience: Theory, review, and hypotheses. Psychology \& Health, 19, 385-405.

Worthington Jr., E. L. \& Wade, N. G. (1999). The psychology of unforgiveness and forgiveness and implications for clinical practice. Journal of Social \& Clinical Psychology, 18, 385-418.

Zechmeister, J. S., Garcia, S., Romero, C. \& Vas, S. N. (2004). Don't apologize unless you mean it: A laboratory investigation of forgiveness and retaliation. Journal of Social \& Clinical Psychology, 23, 532-564. 